\title{
An integrated group decision-making approach considering uncertainty conditions
}

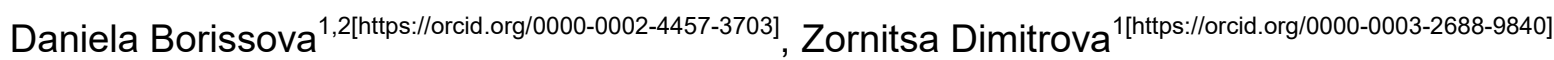 \\ ${ }^{1}$ Institute of Information and Communication Technologies at the Bulgarian Academy of Sciences, \\ Sofia, Bulgaria \\ ${ }^{2}$ Laboratory of Telematics at the Bulgarian Academy of Sciences, Sofia, Bulgaria
}

\begin{abstract}
The management of business information processes needs effective decisionmaking models. That means to involve different methods, techniques, and principles to improve competitiveness and to achieve the planned business results. In this context, the article deals with the problem of group decision-making under uncertain conditions. To cope with such problems some well-known optimization strategies of Wald, Laplace, Hurwitz, and Savage are modified to take into account the experts' opinions with different importance when forming the final group decision. Numerical testing is based on a case study for CRM software selection. The results are discussed based on the proposed models under two different cases derived from the case study. The conducted numerical testing of the proposed models demonstrates their applicability to cope simultaneously with multiple experts' evaluations and uncertainty conditions.
\end{abstract}

Keywords: Group decision-making, uncertainty conditions, cost-benefit estimation, Wald's, Laplace's, Hurwitz's, Savage's criteria

\section{Introduction}

Standing dynamics in the global world is the premise with which managers face every day and need to make decisions to solve different problems. The COVID-19 pandemic forced companies to move quickly to shift to remote working and to build new business models to reflect the new technology capabilities and users' needs. This could be done with the important role of chief information officer [1]. Now, the companies need to determine how to keep and to improve the new business models considering the changing customer demands and ongoing economic uncertainty. To cope with the complex decision-making process, it is necessary to involve multi-disciplinary teams with different qualification area [2]. Involving experts with different expertise will contribute to the credibility of the group decision-making considering different points of view [3]. On the other hand, considering the problems in real life and human judgment are in most cases unclear and cannot be represented by fixed values. Therefore, it is necessary to use different approaches to overcome such conditions. The Bellman-Zadeh approach with some of the well-known optimization strategies proposed by Wald, Laplace, Hurwitz, and Savage could be used to cope with problems under information uncertainty [4]. A systematic review of expert judgement for dependence in probabilistic modelling is presented [5].

The management of operational processes require proper modelling of planned analytical applications to support business in specifics tasks processing [6]. To be successful every company from the large organizations to family businesses needs well-planned information strategy [7]. Therefore, many different approaches are proposed to cope with different practical problems such as effective management in streetlight modernization [8], decision making in publishing sector [9] and selection of supplier under public procurement 
[10], for doing business [11] and business management [12], different real-life applications with time series forecasting [13], etc. The development of business information systems should be based on innovative models and computational intelligence methods. In addition, the development of intelligent software needs to meet the principles of agile software development [14]. Depending on particular domain area different specifics and some common business processes could be recognized from portfolio risk optimisation to loT systems $[15,16]$. In order for a better understanding of the business process maturity models, a systematic literature review is proposed [17]. This is also in line with the policy of the European Data Strategy aiming at the development of an attractive, secure and dynamic data economy. All of these should be an underling of the principles of findability, accessibility, interoperability, and reusability. These principles could be applied also to other digital objects, e.g. algorithms, tools, and workflows, that led to that data, as all these elements must be available to ensure transparency, reproducibility and reusability $[18,19]$.

In the context of digital transformation, a new perspective of digital entrepreneurship driven by the concepts of digital transformation and entrepreneurship is proposed. The authors identify several significant relationships between three categories of digital transformation as technology readiness (ICT investments), digital technology exploration (research and development) and digital technology exploitation (patents and trademarks) that are essential key for business management [20]. Here should be noted also the important role of government to enhance digital transformation in a small service business [21]. The authors show interesting links between business models and business performance. They found correlations between innovation and sustainability, revealing that digital transformation tools contribute over the long-term to the value creation process [22]. Management of business process relies on different methods, techniques and principles aiming to achieve higher business results and competitiveness. The proper methodology for market structures analysis with possibilities for ranking could contribute to business process improvement too $[23,24]$. The success of both business process management and digital innovation could be done by the active role of chief information officers and information technology executives [25].

Business process management requires the use of optimization in order to improve operational efficiency [26]. In addition, in the digital age, businesses need to be not only flexible but also responsive to market conditions. Therefore, it is necessary to provide new levels of optimization of business processes. However, this cannot be achieved only through classical planning techniques and it is necessary to develop new approaches that cover various aspects of the business environment, including the conditions of uncertainty in strategic planning. That is why contemporary business process systems need new methods for thinking about intelligent processes in ways that build on the concepts of collaboration, addictiveness, and awareness [27].

The main paradigm of decision-making is the subjective human factor that cannot be avoided as the decisions are made by humans. Therefore, any attempt to overcome such subjectivism is to be encouraged to reduce bigger and wrong decisions with subsequent accidents $[28,29]$. The usage of group decision-making aims to reduce the subjectivism by involving more experts from different domain area. To get the group decision, it needs to involve proper mathematical models with the ability to aggregate individual preferences of DMs into the final group decision. This means that some quantitative evaluations of the alternatives are to be done concerning predefined criteria. Besides these evaluation criteria need to be further evaluated regarding their importance by each expert.

\section{Integrated Group Decision Making Approach for Evaluation and Choice under Uncertainty Conditions}

During the decision-making process, a set of different alternatives with very different likely consequences must be analysed, i.e. presence of uncertainty in decision making. When making decisions in conditions of uncertainty, it is assumed that the DM has an idea of the 
goals to be achieved, but the information about the alternatives and future events is incomplete. In the decision-making process, the preferences of DM can be represented by a utility function $f(a)$ over a set of alternatives $A=\left\{a_{1}, a_{2}, \ldots, a_{m}\right\}$ in different states $S=$ $\left\{s_{1}, s_{2}, \ldots, s_{n}\right\}$.

\section{Algorithm for Evaluation Considering Different Criteria to Cope with Uncertainty Conditions}

The proposed decision-making algorithm for evaluation considering different criteria to cope with uncertainty conditions is shown in Fig. 1.

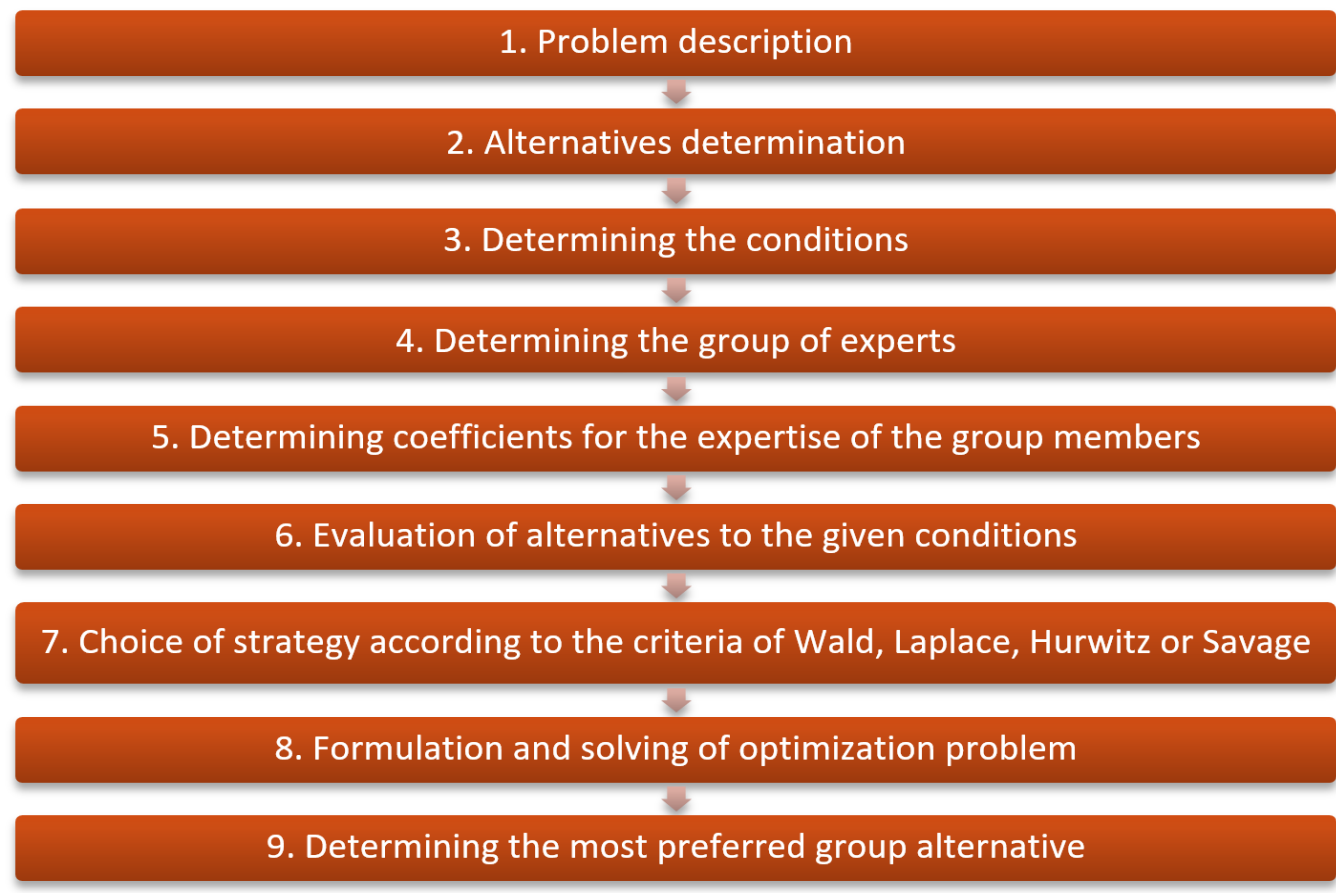

Figure 1. Algorithm for group decision-making under uncertainty conditions.

The first stage for the implementation of group decision-making in the conditions of uncertainty is related to the description of the specific problem for choosing an alternative. Stage 2 is related to identifying the acceptable alternatives appropriate to the problem at hand. At Stage 3, the possible states of the environment in which the identified alternatives are implemented are determined. Depending on the specifics of the identified problem, a group of experts capable of evaluating the alternatives is selected on stage 4 . Once the group of experts are determined, the respective weighting coefficients are to be determined in accordance with the expertise and the degree of importance of each DM in the group. Stage 6 is related to the process of alternatives assessment toward identified conditions and to the point of view of each expert in the group. To cope with uncertainty at stage 7 , one of the well-known Wald's, Laplace's, Hurwitz's or Savage's criteria is to be chosen to determine the optimal strategy. Next, a corresponding optimization task for choosing an alternative in the conditions of group decision making is to be formulated and solved on stage 8 . On the last stage 9, the most preferable group alternative is determined.

To realize the group decision-making under uncertainty conditions it is needed to formulate mathematical models able to aggregate experts' evaluation with respect to the selected criterion to overcome uncertainty. 


\section{Mathematical Models for Group Decision-Making Considering Uncertainty}

As a quantitative analytical tool, revenue and expenditure analysis can be used to support decision making [30]. In this case, a utility function is formulated based on the estimation of expected revenues and expenses. In the general case, the utility function is:

$$
C B E=\frac{\text { Costs }}{\text { Benefits }}
$$

The overall Costs could be expressed as a sum of different aspects from purchasing to installation and maintenance. The Benefits can be expressed by revenue related to some activities that contribute to the reducing labour costs, automated processes, etc.

Using the generalized utility function (1) and the mention above criteria to cope with uncertainty, four modified group decision-making models are formulated as follows:

- Group decision-making model based on Wald's criterion (strategy):

$$
\operatorname{maximin} \sum_{i=1}^{M} \sum_{k=3}^{K} \lambda^{k} C B E_{i j}^{k}
$$

subject to

$$
\sum_{k=1}^{K} \lambda^{k}=1
$$

where $C B E_{i j}^{k}$ expresses evaluation of the $i$-th alternative toward $j$-state from $k$-expert, $\lambda^{k}$ represent the importance of particular expert by corresponding weighted coefficient for the expertise.

Wald's principle is based on a pessimistic view and conservative moderation. It consists of the fact that for any of the chosen strategies, the objective circumstances will always represent the most unfavourable situation. For each alternative solution, the worst outcome can be determined.

- Group decision-making model based on Laplace's criterion (strategy):

$$
\max \left(\sum_{i=1}^{M} \sum_{k=1}^{K} \frac{\lambda^{k} C B E_{i j}^{k}}{M}\right)
$$

subject to relation (3).

According to the Laplace's criterion, when is no other additional information all possible states are considered equally probable.

- Group decision-making model based on Hurwitz's criterion (strategy):

$$
\max \left\{\alpha \max \sum_{i=1}^{M} \sum_{k=1}^{K} \lambda^{k} C B E_{i j}^{k}+(1-\alpha) \min \sum_{i=1}^{M} \sum_{k=1}^{K} \lambda^{k} C B E_{i j}^{k}\right\}
$$

subject to relation (3).

where $\alpha$ is the coefficient of optimism $(0<\alpha<1)$.

The value of the coefficient $\alpha=0$ corresponds to environment considered to be completely antagonistic, $\alpha=0.5$ corresponds to an equivalent environment (neither antagonistic nor friendly) and at a value of $\alpha=1$, the medium is the most favorable. In essence, the Hurwitz's criterion is a simplified version of the Laplace principle, namely - with certain probabilities of the individual states, the arithmetic mean of the results of the best decisions is taken.

- Group decision-making model based on Savage's criterion (strategy):

$$
\operatorname{minimax} \sum_{i=1}^{M} \sum_{k=1}^{K} \lambda^{k} R_{i j}^{k}
$$

subject to relation (3) and one more relation

$$
\forall i=1,2, \ldots M:\left(\forall k=1,2, \ldots M: R_{i j}^{k}=\left|C B E_{i j}^{k}-\max C B E_{i j}^{k}\right|\right)
$$


where $R_{i j}^{k}$ represent the regret as a result of opportunity loss if $A_{i}$ is chosen and state $S_{j}$ happens in accordance to $k$-th expert point of view.

It is possible to use different ranges for the coefficients that express the importance of DMs opinions according to the particular problem, background and expertise, for example between 0 and 10 or range between 1 and 100. In this situation, a proper normalization has to be done to get compatibility between dimensionless weighted coefficients $\left(\lambda^{k}\right)$ and the $C B E$.

\section{Numerical Testing}

Numerical testing of the problem of group decision-making in conditions of uncertainty is made for a specific example of choosing specialized software. Among a variety of specialized systems for customer relationship management (CRM) software, a subset of suitable alternatives with similar characteristics are identified. Due to the dynamism of the economic environment, the companies' prospects can be generally represented by three possible situations: increasing revenues, reducing revenues, or maintaining the current state. Therefore, the subject of the decisions is in the conditions of uncertainty, as the possible situations are known, but it is not known which of them will come true and there is no information about the probabilities for the realization of these situations. The selection of the most appropriate software system could be made through costs benefit analysis as a tool to determine whether the investment decision is good taking into account the mention above possible situations. For the goal, the following representation of costs-benefit relation is formulated:

$$
C B E=\frac{\text { Costs }}{\text { Benefit }}=\frac{C_{a c}+C_{\text {cust }}+C_{\text {inst }}+C_{\text {test }}+C_{\text {staff }}+C_{\text {proc }}+C_{\text {file }}+C_{u n}+C_{l c}}{R_{\text {lcr }}+R_{\text {stock }}+R_{l c}+R_{a p}}
$$

The overall Costs are expressed as a sum of the costs of product acquisition $\left(C_{a c}\right)$, customization $\left(C_{\text {cust }}\right)$, installation $\left(C_{\text {inst }}\right)$, post-installation testing $\left(C_{\text {test }}\right)$, staff training $\left(C_{\text {staff }}\right)$, file conversion $\left(C_{\text {file }}\right)$, uninstallation of the old system $\left(C_{u n}\right)$, and loyalty policy $\left(C_{l c}\right)$. Revenue is related to labor costs reducing $\left(R_{l c r}\right)$, reducing the need to maintain the stock $\left(R_{\text {stock }}\right)$, improved reliability through a new policy for loyal customers $\left(R_{l c}\right)$, the use of more automated processes $\left(R_{a p}\right)$.

Thus, the use of cost-benefit analysis makes it possible to determine the value of CBE as a result of the realization of certain situations.

For the purpose of a small company, it is needed need to make a decision to implement new CRM software. The higher management together with the chief information officer determined 3 suitable alternatives among those the selection should be done. In addition, three experts are also determined to conduct the evaluation. These experts are as follows: an expert in the database (E-2), one end-user of CRM (E-1), one expert from the IT support team (E-3).

The CBEs of the three CRM software systems (alternatives) are done by using the above relation and taking into account the possible three situations for increase, decrease, and maintain the current state of the company as shown in Table 1. 
Table 1. Modified matrix for group decision making under the conditions of uncertainty.

\begin{tabular}{|c|c|c|c|c|c|}
\hline \multirow{2}{*}{$\begin{array}{c}\text { Experts } \\
\text { (DMs) }\end{array}$} & \multirow{2}{*}{$\begin{array}{c}\text { Weighted coefficient } \\
\text { for DMs' expertise }\end{array}$} & \multirow{2}{*}{ Alternatives } & \multicolumn{3}{|c|}{ Conditions } \\
\cline { 3 - 6 } & & Increase & Reduction & Unchanged \\
\hline \multirow{3}{*}{ E-1 } & \multirow{3}{*}{0.25} & $\mathrm{~A}-1$ & 0.52 & 0.83 & 0.75 \\
\cline { 3 - 6 } & & $\mathrm{A}-2$ & 0.53 & 0.86 & 0.72 \\
\cline { 3 - 6 } & & $\mathrm{A}-3$ & 0.54 & 0.84 & 0.71 \\
\hline \multirow{3}{*}{$\mathrm{E}-2$} & \multirow{3}{*}{0.35} & $\mathrm{~A}-1$ & 0.56 & 0.87 & 0.78 \\
\cline { 3 - 6 } & & $\mathrm{A}-2$ & 0.55 & 0.80 & 0.74 \\
\cline { 3 - 6 } & & $\mathrm{A}-3$ & 0.58 & 0.81 & 0.72 \\
\hline \multirow{3}{*}{$\mathrm{E}-3$} & \multirow{3}{*}{0.40} & $\mathrm{~A}-1$ & 0.62 & 0.85 & 0.72 \\
\cline { 3 - 6 } & & $\mathrm{A}-2$ & 0.60 & 0.88 & 0.76 \\
\cline { 3 - 6 } & & $\mathrm{A}-3$ & 0.61 & 0.82 & 0.70 \\
\hline
\end{tabular}

The first two columns of Table 1 contain the weighted coefficients about the importance of each expert (DM) from the formed group.

Using the data from Table 1 and defined group decision-making models based on the Wald, Laplace, and Hurwitz criteria, corresponding tasks are formulated and solved. Savage's criterion requires drawing up a regret matrix. The values of the elements of this matrix represent the losses due to missed opportunities.

The calculation is performed according to the point of view of the DMs from the group according to the following formula:

$$
R_{i j}^{k}=\left|C B E_{i j}^{k}-\max C B E_{i j}^{k}\right|
$$

The values of regret resulting from lost profits are shown in Table 2.

Table 2. Regret matrix as result of opportunity loss.

\begin{tabular}{|c|c|c|c|c|}
\hline \multirow{2}{*}{$\begin{array}{c}\text { Experts } \\
\text { (DMs) }\end{array}$} & Alternatives & \multicolumn{3}{|c|}{ Regret as result of opportunity loss } \\
\hline \multirow{3}{*}{ E-1 } & A-1 & 0.02 & 0.03 & 0 \\
\cline { 2 - 5 } & A-2 & 0.01 & 0 & 0.03 \\
\cline { 2 - 5 } & A-3 & 0 & 0.02 & 0.04 \\
\hline \multirow{3}{*}{ E-2 } & A-1 & 0.02 & 0 & 0 \\
\cline { 2 - 5 } & A-2 & 0.03 & 0.07 & 0.04 \\
\cline { 2 - 5 } & A-3 & 0 & 0.06 & 0.06 \\
\hline \multirow{3}{*}{ E-3 } & A-1 & 0 & 0.03 & 0.04 \\
\cline { 2 - 5 } & A-2 & 0.02 & 0 & 0 \\
\cline { 2 - 5 } & A-3 & 0.01 & 0.06 & 0.06 \\
\hline
\end{tabular}

The described above problem is numerically tested for two cases that express different combination between DMs opinions importance as shown in Table 3.

Table 3. Two cases for the expertise of group members.

\begin{tabular}{|c|c|c|c|}
\hline \multirow{2}{*}{ Cases } & \multicolumn{3}{|c|}{ Weighted coefficient for the experts' expertise } \\
\cline { 2 - 4 } & $\lambda^{\mathbf{1}}$ for E-1 & $\lambda^{\mathbf{2}}$ for E-2 & $\lambda^{\mathbf{3}}$ for E-3 \\
\hline Case-1 & 0.25 & 0.35 & 0.40 \\
\hline Case-2 & 0.34 & 0.46 & 0.20 \\
\hline
\end{tabular}

The Case-1 illustrates the scenario where as the most important is taken on the expert E-1 with weight of 0.4 , closely followed by expert E-2 with a weight of 0.35 and less important of 
the expert E-1 by a weight of 0.25 . The Case-2 represents the experts' importance in the following order E-2, followed by E-1 and finally E-3 (see Table 3).

\section{Results and Discussion}

The formulated four models for group decision-making are based on criteria behind a certain strategy for dealing with uncertainty. Therefore, each of the models can be considered as a model of the respective specific strategy. The results obtained from solving the respective optimization tasks based on the proposed models for group decision-making, taking into account two scenarios for the importance of DM's opinions (Table 3) are illustrated in Fig. 2.

When the Wald's criterion is used, the obtained solutions identified the alternative A-3 as the most preferred in both cases of weights for the experts in the group. Choosing this strategy, the worst possible consequences of each strategy are considered, choosing the least bad or the best of them, according to the point of view of the experts in the group. The choice of this strategy determines the worst possible consequences of each condition and choosing the least bad or the best of them, according to the point of view of the experts in the group.

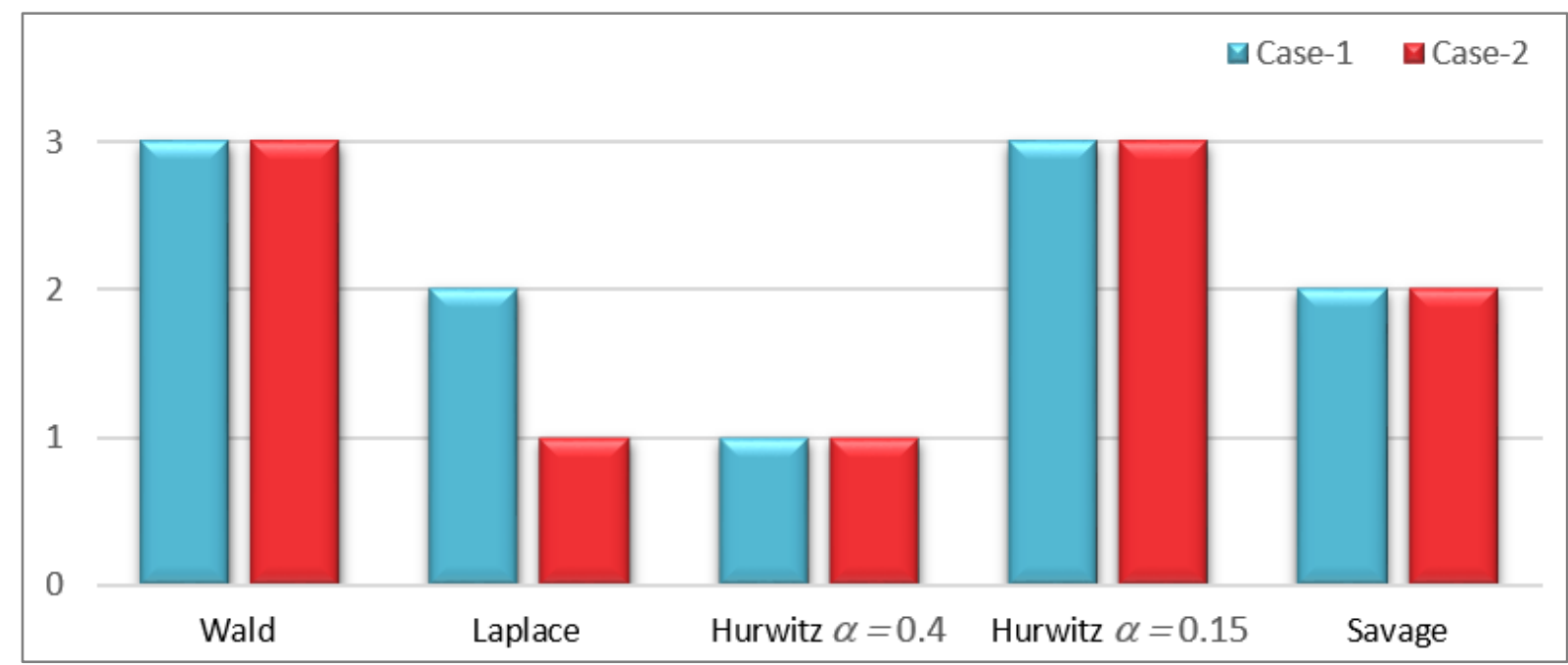

Figure 2. Selected alternative when using different criteria to overcome the uncertainty conditions and different weights for DMs' importance.

According to Laplace's criterion, it is assumed that all objective conditions have the same probability of occurrence due to lack of other grounds. The result is obtained by calculating the arithmetic mean for each strategy, according to the views of the experts in the group and the determined weighting factors, choosing the strategy with the highest score. When using the first combination of weights for experts by Case-1 $\left(\lambda^{1}=0.25 ; \lambda^{2}=0.35 ; \lambda^{3}=0.40\right)$ the solution determines as the most preferred alternative $A-2$, while in the second combination of weights noted asCase- $2\left(\lambda^{1}=0.34 ; \lambda^{2}=0.46 ; \lambda^{3}=0.20\right)$ the most preferred alternative is A1.

Unlike Wald's and Laplace's criteria, Hurwitz's criterion requires the determination of the so-called coefficient of optimism, respectively the coefficient of pessimism, which are applied to each strategy, and the choice is determined by the highest result obtained. Two cases corresponding to two different values for the coefficient of optimism ( $\alpha=0.40$ and $\alpha=0.15$ ) is considered. When using both combinations of weights for experts, for the specific example, and a value of the coefficient of optimism $\alpha=0.40$, the choice for the most preferred is the alternative A-1, and when using $\alpha=0.15$, the solution identifies alternative A3 as the most preferred alternative.

The use of Savage's criterion requires the compilation of a regret matrix as a result of lost profits, choosing this alternative whose maximum losses are minimal. In the particular 
numerical experiment for both uses combinations (Case-1 and Case-2) of weights for the opinions of experts, the most preferred alternative is A-2.

According to the Wild and Hurwitz $(\alpha=0.15)$ strategies and particular input data, the final group decision is to chosen alternative A-3 (Fig. 2) in both cases for DMs' opinions importance. If the strategy behind the Savage principle is chosen, the final group decision is to select the alternative A-2 for both cases for DMs' opinions importance, but according to the strategy behind Laplace principle, the importance of DMs' opinions determines alternative A-2 for Case-1 and alternative A-1 for Case-2 (Fig. 2). The alternative A-1 is also preferable group decision in accordance to the Hurwitz strategy and coefficient of optimism $\alpha=0.40$.

As a result of the analysis, it is found that the use of different strategies according to the principles of Wald, Laplace, Hurwitz, Savage, and in combination with the experts' point of views (Case-1 and Case-2), lead to a different selection of group alternative. It is therefore important to choose in advance the most appropriate decision-making strategy in the face of uncertainty. In summary, it can be concluded that the results of numerical testing of the proposed models for group decision-making in conditions of uncertainty show the applicability of the described modifications based on the optimization criteria of Wald, Laplace, Hurwitz, and Savage.

\section{Conclusions}

The article deals with problems related to group decision-making under uncertain conditions. Such problems are in the focus of many different organizations from SME, including small family businesses, universities, and other non-profit organizations. Due to nowadays dynamic in different aspects of business processes, organizations are facing a variety of decision-making problems with different degrees of uncertain conditions. For the goal, the well-known criteria able to cope with uncertainty like Wald, Laplace, Hurwitz, and Savage are modified to be able to integrate the points of view of experts with different importance when forming final group decision. This is realized by introducing weighted coefficients assigned to each expert. The advantage of such an approach is the possibility to take into account the experts' opinions accordingly to their expertise, background, and closeness to the particular decision-making problem. A drawback of the proposed algorithm could be considered the subjective factor of DM who determines the weighted coefficients of the experts within the group.

The major contributions of the article are related to modified optimization strategies of Wald, Laplace, Hurwitz, and Savage to consider experts' opinions with different importance. These opinions are based on the usage of the calculated value for costs benefits ratio for each particular problem. The components that make up the costs and benefits in case of implementation of a software system are identified, involved in determining the value of the cost-benefit ratio. The proposed approach is applied in the selection of CRM software. It is shown that the final group decision depends not only on the used strategy according to the principles of Wald, Laplace, Hurwitz, and Savage, but is influenced also by the introduced weighed coefficients expressing the experts' opinion importance when aggregating the final group decision.

Future developments concern the development of different models for more precise and objective estimations of the weights for experts' opinions in an aggregation of a final group decision. Another perspective direction is related to the use of a different scale for the alternatives estimation that differs from the used cost-benefit estimation ratio. 


\section{References}

1. V. Shalamanov, V. Sabinski, T. Georgiev, "Optimization of the chief information officer function in large organizations", Information \& Security, vol. 46, no. 1, pp. 13-26, 2020, doi: https://doi.org/10.11610/isij.4601.

2. D. Borissova, Z. Dimitrova, V. Dimitrov, "How to support teams to be remote and productive: Group decision-making for distance collaboration software tools", Information \& Security, vol. 46, no. 1, pp. 36-52, 2020, doi: https://doi.org/10.11610/isii.4603.

3. D. Borissova, "A group decision making model considering experts competency: An application in personnel selections", Comptes rendus de l'Academie Bulgare des Sciences, vol. 71, no. 11, pp. 1520-1527, 2018.

4. P. Ekel, J.S.C. Martini, R.M. Palhares, "Multicriteria analysis in decision making under information uncertainty", APPL MATH COMPUT, vol. 200, no. 2, pp. 501-516, 2008, doi: https://doi.org/10.1016/j.amc.2007.11.024.

5. C. Werner, T. Bedford, R.M. Cooke, A.M. Hanea, O. Morales-Napoles, "Expert judgement for dependence in probabilistic modelling: A systematic literature review and future research directions," EUR J OPER RES, vol. 258, no. 3, pp. 801-819, 2017, doi: https://doi.org/10.1016/i.ejor.2016.10.018.

6. C. Hrach, R. Alt, "Configuration approach for analytical service models - development and evaluation", in: 2020 IEEE 22nd CONF BUS INFORM, Antwerp, Belgium, 2020, pp. 260-269. https://doi.org/10.1109/CBI49978.2020.00035.

7. M. Kamariotou, F. Kitsios, "Information Systems Planning and Success in SMEs: Strategizing for IS", in: BIS 2019, LNBIP, vol. 353, 2019, pp. 397-406, https://doi.org/10.1007/978-3-030-20485-3

8. D. Borissova, P. Cvetkova, I. Garvanov, M. Garvanova, "A framework of business intelligence system for decision making in efficiency management", in: CISIM'2020, LNCS, vol. 12133, 2020, pp. 111-121. https://doi.org/10.1007/978-3-030-47679-3 10.

9. D. Borissova, N. Keremedchieva, D. Keremedchiev, "Business intelligence approach to support decision making in publishing sector", MIPRO, pp. 1532-1537, 2020, doi: https://doi.org/10.23919/MIPRO48935.2020.9245424.

10. D. Korsemov, D. Borissova, I. Mustakerov, "Group decision making for selection of supplier under public procurement", in: ICT Innovations 2018, COMM COM INF SC, vol. 940, 2018, pp. 51-58. https://doi.org/10.1007/978-3-030-00825-3 5.

11. D. Borissova, D. Korsemov, I. Mustakerov, "Multi-criteria decision making problem for doing business: Comparison between approaches of individual and group decision making", in: CISIM'2019, LNCS, vol. 11703, 2019, pp. 385-396. https://doi.org/10.1007/978-3-030-28957-7 32.

12. I. Stankov, G. Tsochev, "Vulnerability and protection of business management systems: Threats and challenges. Problems of Engineering Cybernetics and Robotics, vol. 72, pp. 29-40, 2020, https://doi.org/10.7546/PECR.72.20.04.

13. R. Ketipov, G. Kostadinov, P. Petrov, I. Zankinski, T. Balabanov, "Genetic algorithm based formula generation for curve fitting in time series forecasting implemented as mobile distributed computing", in: ADV HIGH PERF COM 2019, STUD COMP INTELL, vol. 902, 2021, pp. 40-47. https://doi.org/10.1007/978-3-030-55347-0 4.

14. M. Perkusich, L. Chaves e Silva, A. Costa, F. Ramos, R. Saraiva, A. Freire, E. Dilorenzo, E. Dantas, D. Santos, K. Gorgonio, H. Almeida, A. Perkusich, "Intelligent software engineering in the context of agile software development: A systematic literature review", INFORM SOFTWARE TECH, vol. 119, no. 106241, 2020, doi: https://doi.org/10.1016/j.infsof.2019.106241.

15. K. Stoyanova, V. Guliashki, "Two-stage portfolio risk optimisation based on MVO model", International Journal of Reasoning-based Intelligent Systems, vol. 12, no. 1, pp. 70-79, 2020, doi: https://dx.doi.org/10.1504/IJRIS.2020.105011.

16. A. Vodyaho, R. Yoshinov, N. Zhukova, A.M. Thaw, A. Saddam Ahmed, "Fog oriented model for data collection in the networks of mobile devices", in: 2020 IEEE 10th INT 
CONF INTELL SYST, Varna, Bulgaria, 2020, pp. 421-425.

https://doi.org/10.1109/IS48319.2020.9200138.

17. A. Tarhan, O. Turetken, H.A. Reijers, "Business process maturity models: A systematic literature review", INFORM SOFTWARE TECH, vol. 75, pp. 122-134, 2016, doi: https://doi.org/10.1016/j.infsof.2016.01.010.

18. L. Liu, W. Li, N.R. Aljohani, M.D. Lytras, S.-Ul Hassan, R. Nawaz, "A framework to evaluate the interoperability of information systems - Measuring the maturity of the business process alignment", INT J INFORM MANAGE, vol. 54, no. 102153, 2020, doi: https://doi.org/10.1016/j.ijinfomgt.2020.102153.

19. A.-L. Lamprecht, et al., "Towards FAIR principles for research software," Data Science, vol. 3, no.1, pp. 37-59, 2020, doi: https://doi.org/10.3233/DS-190026.

20. V. Jafari-Sadeghi, A. Garcia-Perez, E. Candelo, J. Couturier, "Exploring the impact of digital transformation on technology entrepreneurship and technological market expansion: The role of technology readiness, exploration and exploitation", J BUS RES, vol. 124, pp. 100-111, 2021, doi: https://doi.org/10.1016/j.jbusres.2020.11.020.

21. C.-L. Chen, Y.-C. Lin, W.-H. Chen, C.-F. Chao, H. Pandia, "Role of government to enhance digital transformation in small service business", Sustainability, vol. 13, no. 3, 2021, doi: https://doi.org/10.3390/su13031028.

22. A. Di Vaio, R. Palladino, A. Pezzi, D.E. Kalisz, "The role of digital innovation in knowledge management systems: A systematic literature review", J BUS RES, vol. 123, pp. 220-231, 2021, doi: https://doi.org/10.1016/j.jbusres.2020.09.042.

23. I. Petrov, "Improving the methodology of market structures analysis with innovative concepts for phase-structure states and set concentration index", Economic Alternatives, vol. 1, pp. 5-15, 2016.

24. I. Mustakerov, D. Borissova, "Investments attractiveness via combinatorial optimization ranking", International Journal of Management Science and Engineering vol. 7, no. 10, pp. 230-235, 2013, doi: https://doi.org/10.5281/zenodo.1088218.

25. A. Van Looy, "A quantitative and qualitative study of the link between business process management and digital innovation", INFORM MANAGE, vol. 58, no. 2, 103413 (2021). https://doi.org/10.1016/j.im.2020.103413.

26. M. Camargo, M. Dumas, O. Gonzalez-Rojas, "Automated discovery of business process simulation models from event logs", DECIS SUPPORT SYST, vol. 134, 113284, 2020, doi: https://doi.org/10.1016/j.dss.2020.113284.

27. H. Kir, N. Erdogan, "A knowledge-intensive adaptive business process management framework", Information Systems, vol. 95, 101639, 2021, doi: https://doi.org/10.1016/j.is.2020.101639.

28. F. Klapproth, "Time and decision making in humans", Cognitive, Affective, \& Behavioral Neuroscience, vol. 8, pp. 509-524, 2008, doi: https://doi.org/10.3758/CABN.8.4.509.

29. R. Moura, C. Morais, E. Patelli, J. Lewis, M. Beer, "Human factors influencing decision-making: tendencies from first-line management decisions and implications to reduce major accidents", in: ESREL'2017, Proc. of International Conference on Engineering Sciences and Technologies, 2017, pp. 69-34. doi: 10.1201/9781315210469-34

30. A. Boardman, D. Greenberg, A. Vining, D. Weimer, "Cost benefit analysis: Concepts and practice", The Pearson Series in Economics, 4th edn., Pearson, 2010. 